\title{
Antiferromagnetic Order in the Half-Heusler Phase TbPdBi
}

\author{
O. PavlosiuK*, M. Kleinert, P. Wiśniewski and D. Kaczorowski \\ Institute of Low Temperature and Structure Research, Polish Academy of Sciences,
}

Okólna 2, 50-422 Wrocław, Poland

\begin{abstract}
Single crystals of $\mathrm{TbPdBi}$, a representative of the group of half-Heusler bismuthides, were studied by means of magnetic susceptibility, heat capacity, electrical resistivity, magnetostriction and thermal expansion measurements. The compound was characterized as an antiferromagnet with the Néel temperature $T_{\mathrm{N}} \approx 5.3 \mathrm{~K}$. Neutron diffraction experiment confirmed the antiferromagnetic ordering and yielded the propagation vector $\boldsymbol{k}=\left(\frac{1}{2}, \frac{1}{2}, \frac{1}{2}\right)$. Remarkably, this $\boldsymbol{k}$ vector is in accord with the recently developed theory of antiferromagnetic topological insulators.
\end{abstract}

DOI: 10.12693/APhysPolA.133.498

PACS/topics: 75.50.Ee, 75.80.+q, 71.55.Ak, 72.15.Eb

\section{Introduction}

In recent years, rare-earth $(R)$-based half-Heusler phases crystallizing with the MgAgAs-type crystal structure (space group $F-\overline{4} 3 m$ ) have attracted much attention owing to their plausible topologically non-trivial electronic structures [1]. In particular, these materials were theoretically predicted to exemplify an exciting novel category of antiferromagnetic topological insulators [2, 3]. Most recently, the prediction was verified experimentally for GdPtBi that was characterized as an antiferromagnetic Weyl semimetal [4].

According to the literature data [5-11], the halfHeusler bismuthides $R \mathrm{PdBi}$ order magnetically with rather low Néel temperatures. Remarkably, some of them exhibit coexistence of superconductivity and antiferromagnetism [6, 7, 9-11] In this paper, we report on the low-temperature thermodynamic and transport properties of single-crystalline TbPdBi, supplemented by neutron diffraction data

\section{Experimental details}

Single crystals of TbPdBi were obtained in a two-stage process. First, a polycrystalline sample was prepared by arc-melting the constituents. Then, the ingot was powdered, mixed with Bi flux in the ratio 1:15, put into an alumina crucible and sealed in evacuated quartz ampule. The tube was heat treated in a way described in Ref. [6]. The so-synthesized single crystals were characterized by X-ray diffraction (XRD) on an Oxford Diffraction X'calibur four-circle diffractometer.

Magnetic properties were determined using a Quantum Design MPMS-XL magnetometer. Electrical transport and heat capacity measurements were carried out employing a Quantum Design PPMS platform. Magnetostriction and thermal expansion was measured in the PPMS using a miniature capacitance dilatometer [12].

*corresponding author; e-mail: o.pavlosiuk@int.pan.wroc.pl

Neutron diffraction experiment was performed at 2 and $10 \mathrm{~K}$ on a two-axis neutron diffractometer in Laboratoire Léon Brillouin, CEA-Saclay, using neutrons of $0.84 \AA$ wavelength.

\section{Results and discussion}

The XRD data analysis confirmed that TbPdBi forms with the MgAgAs-type crystal structure, and the refined lattice parameter $a=6.662 \AA$ is close to that reported in the literature [13].

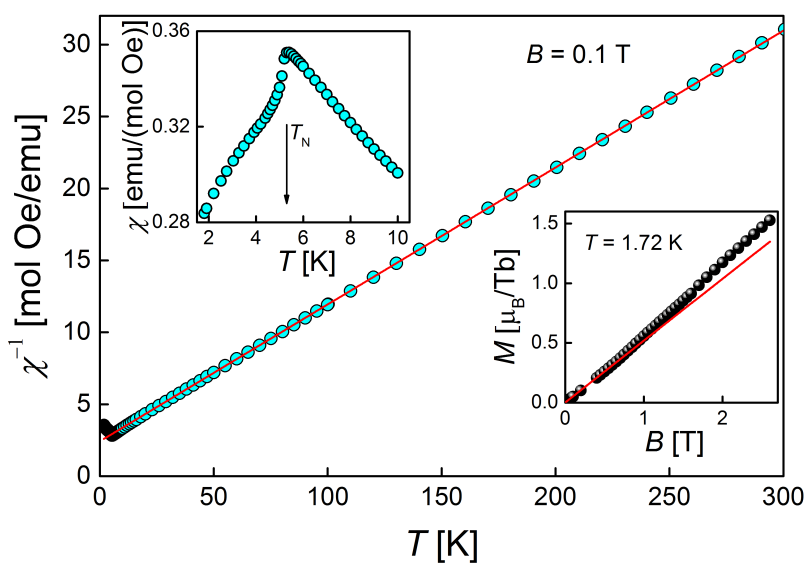

Fig. 1. Temperature dependence of the reciprocal magnetic susceptibility of TbPdBi. Solid line represents the Curie-Weiss fit. Upper inset: the low-temperature magnetic susceptibility data. Arrow marks the Néel temperature. Lower inset: field variation of the magnetization in TbPdBi recorded at $T=1.72 \mathrm{~K}$. Solid line emphasizes a metamagnetic-like behaviour.

Figure 1 displays the magnetic properties of $\mathrm{TbPdBi}$. Above ca. $6 \mathrm{~K}$, the magnetic susceptibility follows the Curie-Weiss law:

$$
\chi^{-1}=\frac{3 k_{\mathrm{B}}\left(T-\theta_{p}\right)}{N_{\mathrm{A}} \mu_{e f f}^{2}}
$$

with the paramagnetic Curie temperature $\theta_{p}=-25.5 \mathrm{~K}$ and the effective magnetic moment $\mu_{e f f}=9.17 \mu_{\mathrm{B}}$. These parameters are similar to those reported in Ref. [11]. The value of $\mu_{\text {eff }}$ is close to the theoretical 
one $9.72 \mu_{\mathrm{B}}$ expected for a free $\mathrm{Tb}^{3+}$ ion. As shown in the upper inset to Fig. 1, $\chi(T)$ exhibits a sharp maximum at $T_{\mathrm{N}}=5.3 \mathrm{~K}$ that signals the onset of antiferromagnetic ordering. Nearly the same value of $T_{\mathrm{N}}$ was reported before [11]. The antiferromagnetic ground state in $\mathrm{TbPdBi}$ is corroborated by the characteristic inflection in the magnetic field variation of the magnetization measured at $T=1.72 \mathrm{~K}$, which occurs near $0.6 \mathrm{~T}$ (see the lower inset to Fig. 1). This feature manifests a metamagnetic-like transition.

As can be inferred from Fig. 2, the antiferromagnetic ordering in TbPdBi manifests itself as distinct anomalies in the temperature variations of the heat capacity and the electrical resistivity. A mean-field type $\lambda$-shaped anomaly in $C(T)$ occurs at $5.1 \mathrm{~K}$, in line with $T_{\mathrm{N}}$ derived from the magnetic data. At the same temperature, $\rho(T)$ exhibits a drop, signalling a decrease in spin-disorder scattering contribution (note the inset to Fig. 2a). In the paramagnetic region, the resistivity of TbPdBi shows a non-monotonic behaviour. Up to about $80 \mathrm{~K}$, the compound behaves like a metal, whereas at higher temperatures its electrical conductivity changes in a manner typical for semiconductors. The overall shape of $\rho(T)$ is similar to that reported for TbPdBi by Nakajima et al. [11], and comparable to the behaviour found for other $R \mathrm{PdBi}$ compounds $[5-10]$.
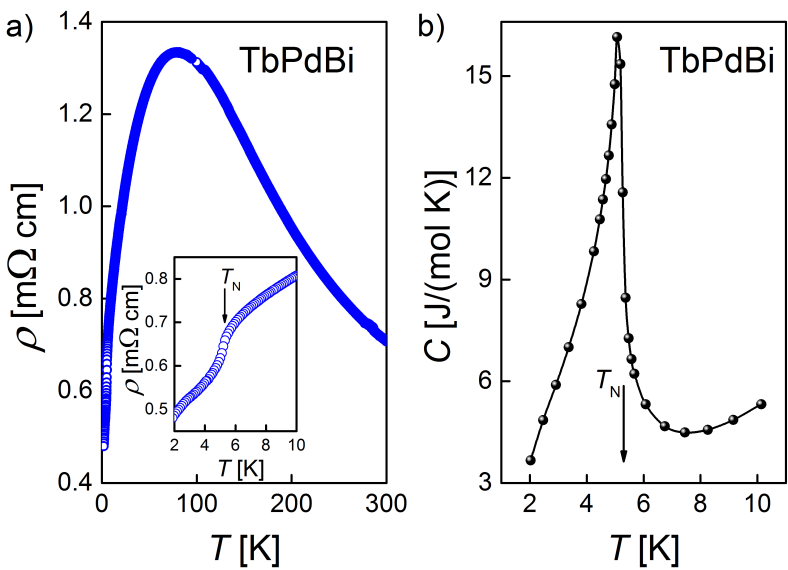

Fig. 2. (a) Electrical resistivity of TbPdBi as a function of temperature. Inset: the low-temperature resistivity data. (b) Low-temperature dependence of the specific heat of TbPdBi. Arrow marks the Néel temperature.

Figure 3 presents the elastic properties of TbPdBi. The temperature-dependent relative sample length change $\Delta L(T) / L_{0}$, where $L_{0}$ corresponds to the sample length at $T=2 \mathrm{~K}$, and $\Delta L=L_{T}-L_{0}$, is shown in the inset to Fig. 3a. The thermal expansion is positive in the entire range of temperature and gradually decreases with lowering temperature down to $T_{\mathrm{N}}$, at which temperature a sudden drop in $\Delta L / L_{0}$ occurs. The order of magnitude of $\Delta L / L_{0}$ of our sample is comparable to that reported for HoPdBi [10]. As shown in Fig. 3a, in external magnetic fields, the anomaly in $\Delta L(T) / L_{0}$ slightly moves to lower temperatures with increasing the field strength, as expected for antiferromagnets. Furthermore, in the ordered state and in fields above the metamagnetic-like transition, the compound exhibits negative thermal expansion, i.e. $\Delta L / L_{0}$ increases with decreasing temperature.
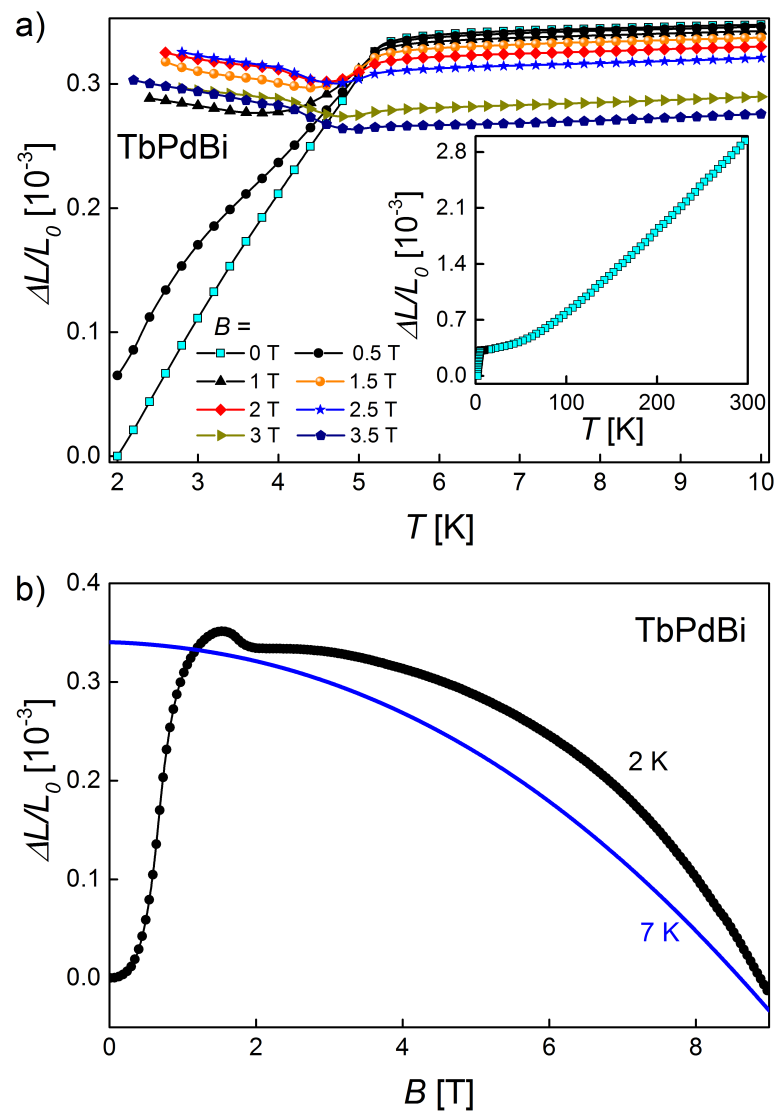

Fig. 3. (a) Relative length change of the sample of $\mathrm{TbPdBi}$ as a function of temperature, measured in different applied magnetic fields. Inset: zero-field temperature variation of the thermal expansion of TbPdBi up to room temperature. (b) Magnetostriction in TbPdBi recorded at $T=2 \mathrm{~K}$ and $7 \mathrm{~K}$.

Figure $3 \mathrm{~b}$ displays the magnetostriction in $\mathrm{TbPdBi}$ measured in the ordered and paramagnetic states. Above $T_{\mathrm{N}}, \Delta L / L_{0}$ smoothly decreases with increasing the magnetic field strength. In contrast, at $T=2 \mathrm{~K}, \Delta L / L_{0}$ first increases sharply in weak fields, goes through a maximum of 0.35 near $1.5 \mathrm{~T}$, makes a clear kink at $B=2 \mathrm{~T}$, and eventually decreases with further increasing magnetic field. The observed complex behaviour of $\Delta L(B) / L_{0}$ originates in the antiferromagnetic character of the ground state in TbPdBi. Specifically, the $\Delta L(B) / L_{0}$ curve exhibits an inflection at the metamagnetic-like transition in $B=0.6 \mathrm{~T}$.

The main result of the neutron diffraction study on single-crystalline $\mathrm{TbPdBi}$ is summarized in Fig. 4. Very Intense Polarized neutron diffractometer, which was used 
for measurements is equipped with position-sensitive detector [14]. This allows to measure simultaneously diffraction intensities in large portions of reciprocal space and easily detect and identify super-structure reflections, especially magnetic ones. The experiment was carried out at temperatures above and below $T_{\mathrm{N}}$. We observed numerous additional reflections in diffraction pattern obtained below $T_{\mathrm{N}}$. Subtraction of the intensities recorded for the $\left(0,0, \frac{3}{2}\right)$ plane of the reciprocal space above $T_{\mathrm{N}}$ from those collected at $T=2 \mathrm{~K}$ gave a differential intensity map which is shown in Fig. 4. The positions of these magnetic Bragg reflections indicate the propagation vector $\boldsymbol{k}=\left(\frac{1}{2}, \frac{1}{2}, \frac{1}{2}\right)$. Remarkably, this $\boldsymbol{k}$ value complies with the theoretical conditions for antiferromagnetic topological insulators [3], which opens a possibility that TbPdBi is another, after GdPtBi and $\mathrm{HoPdBi}$, representative of this exciting novel family of materials $[4,6]$.

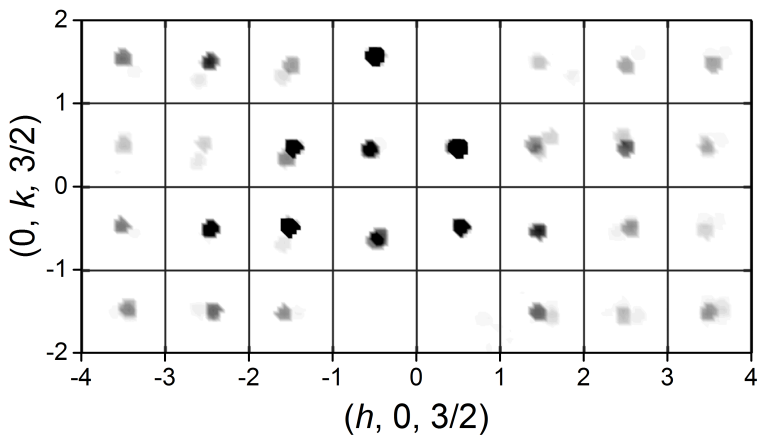

Fig. 4. The $\left(h, k, \frac{3}{2}\right)$ differential (magnetic) neutron diffraction intensity map of TbPdBi.

\section{Conclusions}

The half-Heusler phase $\mathrm{TbPdBi}$ is an antiferromagnet with $T_{\mathrm{N}}=5.3 \mathrm{~K}$, as revealed by magnetic susceptibility, magnetization, electrical resistivity, specific heat, thermal expansion and magnetostriction measurements performed on single-crystalline specimens. The antiferromagnetic ordering was confirmed by neutron diffraction, which yielded the propagation vector $\boldsymbol{k}=\left(\frac{1}{2}, \frac{1}{2}, \frac{1}{2}\right)$. The latter finding rises a possibility that $\mathrm{TbPdBi}$ is another antiferromagnetic Weyl semimetal similar to GdPtBi [24]. To verify this tempting hypothesis, microscopic characterization of the compound by means of angle-resolved photoemission spectroscopy is required.

\section{Acknowledgments}

This research was supported by the National Science Centre of Poland, grant no. 2015/18/A/ST3/00057. The authors are grateful to Arsen Gukasov and Xavier Fabreges for their kind assistance in the neutron diffraction experiment and the data analysis.

\section{References}

[1] S. Chadov, X. Qi, J. Kübler, G.H. Fecher, C. Felser, S.C. Zhang, Nature Mat. 9, 541 (2010).

[2] R.S.K. Mong, A.M. Essin, Phys. Rev. B 81, 245209 (2010).

[3] R.A. Müller et al., Phys. Rev. B 90, 041109(R) (2014).

[4] M. Hirschberger et al., Nat. Mater. 15, 1161 (2016).

[5] O. Pavlosiuk, K. Filar, P. Wiśniewski, D. Kaczorowski, Acta Phys. Pol. A 127, 656 (2015).

[6] O. Pavlosiuk, D. Kaczorowski, X. Fabreges, A. Gukasov, P. Wiśniewski, Sci. Rep. 6, 18797 (2016).

[7] O. Pavlosiuk, P. Wiśniewski, D. Kaczorowski, Acta Phys. Pol. A 130, 573 (2016).

[8] K. Gofryk, D. Kaczorowski, T. Plackowski, A. LeitheJasper, Yu. Grin, Phys. Rev. B 84, 035208 (2011).

[9] Y. Pan et al., Europhys Lett.104, 27001 (2013).

[10] A. M. Nikitin et al., J. Phys.: Condens. Matter 27, 275701 (2015).

[11] Y. Nakajima et al., Sci. Adv. 1, e1500242 (2015).

[12] R. Küchler, T. Bauer, M. Brando, F. Steglich, Rev. Sci. Instrum. 83, 095102 (2012).

[13] M. G. Haase, T. Schmidt, C. G. Richter, H. Block, W. Jeitschko, J. Sol. State Chem. 168, 18 (2002).

[14] A. Gukasov et al., Phys. Procedia 42, 150 (2013). 